\title{
Absorber-Free Quasi-Simultaneous Laser Welding For Microfluidic Applications
}

\author{
Nam-Phong Nguyen ${ }^{1}$, Maximilian Brosda ${ }^{1}$, Alexander Olowinsky ${ }^{1}$ and Arnold Gillner ${ }^{2}$ \\ ${ }^{1}$ Fraunhofer Institute for Laser Technology ILT, Steinbachstraße 15, 52074 Aachen, Germany \\ ${ }^{2}$ Chair for Laser Technology LLT, Steinbachstraße 15, 52074 Aachen, Germany \\ E-mail address: phong.nguyen@ilt.fraunhofer.de
}

\begin{abstract}
Absorber-free laser transmission welding is a novel welding technique which enables joining of polymers with the same optical properties. As no absorber is needed, this method is especially predestined for the encapsulation of transparent polymers for life science applications such as microfluidic devices. Due to the high welding seam densities and lengths of microfluidic devices, it is crucial to keep the thermal stress low in order to avoid the formation of cracks and distortions. On top of that, a melt flow into the channel structures has to be avoided to ensure the correct operation of the device. Therefore, a precise energy deposition into the interface during the welding process has been the subject of research for many years. One promising approach is a quasi-simultaneous welding technique where the laser beam is guided along the welding contour multiple times at high feed rates of up to several $\mathrm{m} / \mathrm{s}$. In the present work, a welding process is developed to encapsulate microfluidic devices by using this technique. In order to avoid heat accumulation, the welding contour was divided in several segments. By defining an irradiation sequence for each segment, a homogenous heat distribution on the sample can be achieved.

DOI: 10.2961/jlmn.2019.03.0009
\end{abstract}

Keywords: laser welding, transparent polymers, microfluidic devices

\section{Introduction}

The growing demands on the quality and complexity of plastic components lead to increasing requirements to the production processes. As a consequence, plastic components consist of several single parts which have to be joined together. In case of high-tech products from the automotive, electronics and medical sectors, welding joints have to be produced in the smallest dimensions while maintaining a high quality. Here, laser transmission welding (LTW) offers unique advantages such as a contactless and highly precise energy input. Therefore, it is possible to create complex welding seam geometries using the spatially and temporally defined energy input $[1,2]$. There are currently two welding techniques available which can be distinguished by the wavelength of the utilised beam source (see Fig. 1).

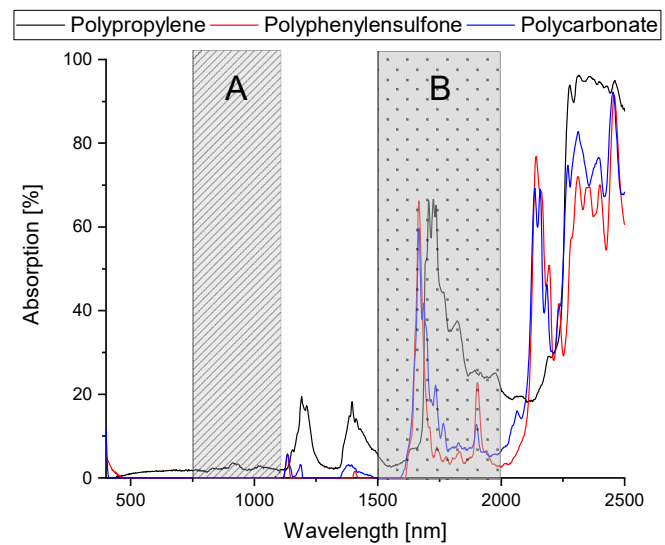

Fig. 1 Absorption curves in the wavelength area of classic (A) and absorber-free (B) LTW
In the first technique, classic LTW, conventional beam sources are used which emit radiation in the wavelength range between $800-1100 \mathrm{~nm}$. As most technical polymers are transparent in this area (see Fig. 1, A), the use of absorbing additives is necessary in order to create a welding seam. In case of a welding process in an overlap configuration, the absorbers are added to the lower joining partner. The laser light is transmitted through the upper joining partner and absorbed at the surface of the lower joining partner. The absorbed radiation is converted into heat which leads to a melting of both joining partners. Here, the area of absorption is always located in the interface of both joining partners $[3,4]$.

By using novel beam sources which emit radiation in the wavelength range between $1500-2000 \mathrm{~nm}$, it is nowadays possible to weld polymers without using absorbing additives (see Fig. 1, B). The reason is that most polymers show a natural absorption behaviour in this range. Hence, it is possible to weld transparent polymers without using absorbing additives [5,6]. Typical beam sources which are used here are thulium fibre lasers $(\lambda=1940 \mathrm{~nm})$. This makes this technique predestined for the encapsulation of multilayer polymeric films for sensitive electronic devices (e.g. OLEDs) or for life science applications such as microfluidic devices $[7,8]$. Several works are available for both butt joint and overlap configuration [3,5,6-9].

In contrast to classic LTW where the heat affected zone (HAZ) is locally confined to the welding interface, the HAZ is vertically extended along the entire cross section in absorber-free LTW (see Fig. 2) [3]. 


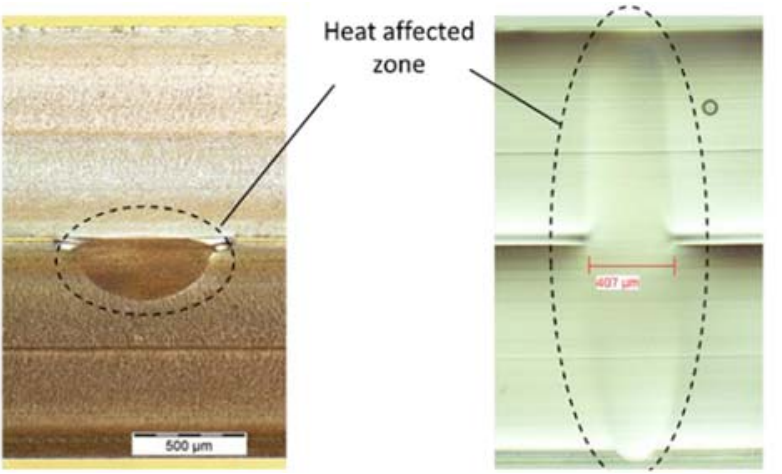

Fig. 2 Different HAZ geometries in classic (left) and absorberfree LTW (right) [10]

The reason for the HAZ geometries are the different absorption mechanisms in both welding techniques. In classic LTW, the laser energy is nearly entirely transmitted in the upper joining partner and absorbed at the surface of the lower joining partner (see Fig. 2, left) [1,2]. Hence, the HAZ is always located in the interface. In absorber-free LTW, the laser energy is absorbed in both joining partners as they have the same optical properties. The intensity progression inside the joining partners can be described by the Beer-Lambert law [3]:

$I(z)=(1-R) * I_{0} * e^{-\alpha * Z}$

where $\mathrm{R}$ is the reflection, $\mathrm{I}_{0}$ is the laser intensity on the surface and $\alpha$ is the absorption coefficient. The optical penetration depth $\delta$ determines the material depth in which the laser radiation decreases to $1 / \mathrm{e}$ (about $37 \%$ ). It is defined as [3]:

$$
\delta=\alpha^{-1}
$$

As the absorption coefficient $\alpha$ of the natural polymer is much lower compared to the optically modified polymer, the optical penetration depth $\delta$ is higher which leads to an absorption inside the whole irradiated material volume $[3,8$, $10]$. The different absorption mechanisms and intensity progressions are shown in Figure 3.

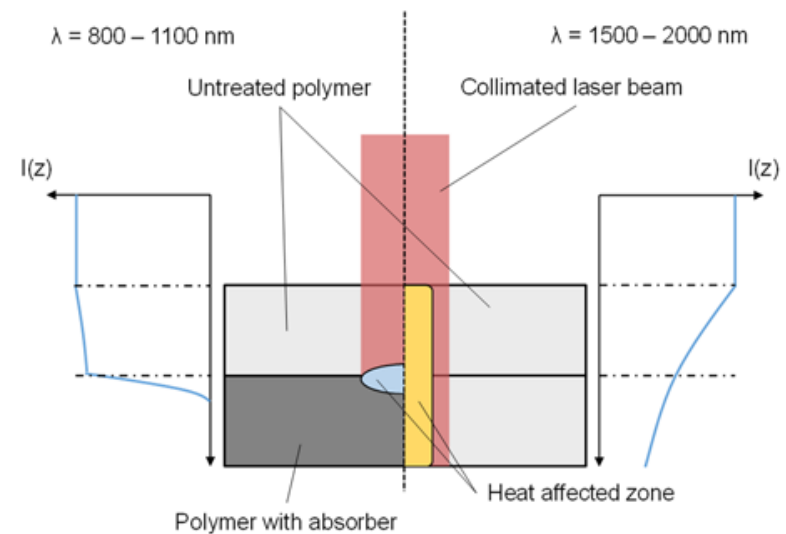

Fig.3 Intensity progression in classic (left) and absorber-free LTW (right).

The lateral expansion of the HAZ in absorber-free LTW leads to high thermal stress. As a result, cracks as well as distortions can occur due to the expansion and contraction of the polymer melt during the welding process. Especially in microfluidic applications where the seam lengths can reach several metres due to the high channel densities, it is crucial to keep the thermal stress low.

Therefore, the reduction of the HAZ in the vertical direction has been the subject of research for many years [3,8,10-12]. Ideally, the HAZ should only be confined in the interface of both joining partners as in classic LTW.

Aden et al. reported on theoretical investigations on the energy input in materials with the same absorption properties $[8,12]$. Here, two opposing effects can be detected. First, the laser intensity $\mathrm{I}_{0}$ decreases due to absorption along the beam propagation direction as shown in equation 1 . The heating rate is therefore the highest at the surface of the upper joining partner. The second effect takes place when the laser beam is focused in the interface between the joining partners. The decreasing beam diameter leads to an increase of the laser intensity until a maximum is reached at the focal plane. However, as these two effects are counteracting each other, the laser beam has to be strongly focused by using optics with a high numerical aperture (NA) in order to overcome the first effect and to selectively heat up the polymer. It could be shown that a selective temperature distribution can be achieved when two conditions are fulfilled [8,12]:

$z_{R} \leq \frac{1}{2 * \alpha}$

$\alpha<\frac{1}{d}$

where $z_{R}$ is the Rayleigh length, $\alpha$ the absorption coefficient and $d$ the thickness of the upper joining partner.

Apart from the presented conditions, the thermal conduction also affects the temperature distribution. Another approach was first introduced by Mamuschkin et al. by using a quasi-simultaneous irradiation technique [3]. Here, the laser beam is moved several times at high feed rates ( $v>1 \mathrm{~m} / \mathrm{s}$ ) leading to a nearly simultaneous heating of the entire welding contour. The high feed rates are achieved by using galvanometric scanning systems. In case of classic LTW, this method is predominantly used due to its' good gap bridging capabilities compared to contour welding as the whole contour is plasticized $[3,13]$. In case of absorber-free LTW, heat is dissipated from the upper and lower surface after each irradiation pass. With increasing number of passes $\mathrm{n}$ and feed rate $\mathrm{v}$, a reduction of the HAZ could be achieved. It could be shown that the vertical extent of the HAZ could be reduced by $30 \%$ compared to contour welding [3].

Within the scope of the present work, the quasi-simultaneous welding technique will be investigated in more detail. First, the influence of the focal position and the number of passes on the HAZ will be analysed. Since high seam densities occur especially in microfluidic components, the influence of the seam distance and the cooling time on the HAZ geometry will be investigated (see chapter 3 ). In chapter 4 , the welding contour will be divided into several segments. The influence of the irradiation order of each segment on the heat distribution will be investigated. The generated process understanding will then be applied to the encapsulation of a microfluidic test component (see chapter 5). 


\section{Experimental setup and analysation of $\mathrm{HAZ}$}

As testing material, polycarbonate (PC) was used (Makrolon $\AA$, Covestro AG, Leverkusen, Germany) with a thickness of $\mathrm{d}=1 \mathrm{~mm}$ and an absorption coefficient of $\alpha=0.103 \mathrm{~mm}^{-1}$. The average surface roughness $R_{a}$ is $0.092 \mu \mathrm{m}$. A thulium fibre laser (IPG Laser GmbH, Burbach, Germany) with a wavelength of $\lambda=1940 \mathrm{~nm}$ was used with a maximum output power $\mathrm{P}=120 \mathrm{~W}$. The laser beam was moved using an IntelliScan 14 (Scanlab GmbH, Puchheim, Germany) and focused using a f-theta lens with a focal length of $100 \mathrm{~mm}$. A clamping device was used to enable a thermal contact between both joining partners as well as a reproducible positioning. The sample holder is made of aluminium which has a heat conductivity $\kappa=235 \mathrm{~W} /(\mathrm{m} * \mathrm{~K})$. The high heat conductivity leads to good heat dissipation during the quasi-simultaneous welding trials. On the other hand, the high reflectance couples the reflected laser beam back into the sample. The experimental setup is shown in Fig.4.

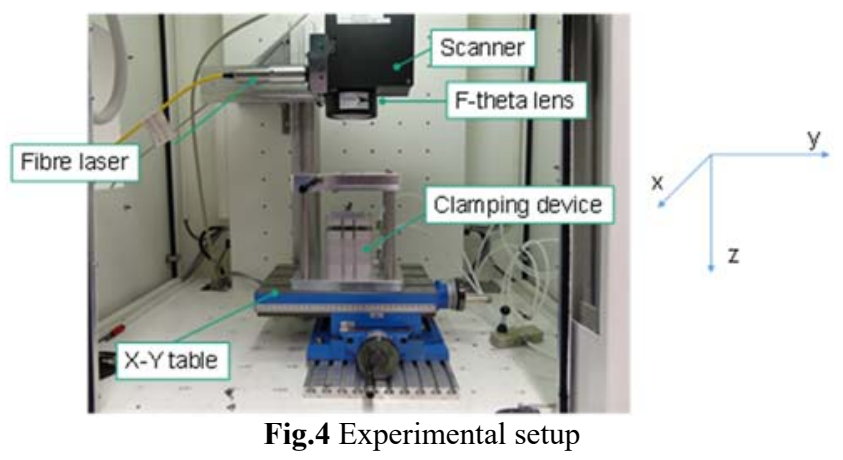

A summary of the laser system and the beam properties can be found in the following table.

Tab.1: Laser system and beam properties

\begin{tabular}{|l|l|l|l|}
\hline \multicolumn{2}{|c|}{ Laser system } & \multicolumn{2}{c|}{ Beam properties } \\
\hline Beam source & IPG TLR-120-1940 & Focal diameter & $90 \mu \mathrm{m}$ \\
\hline Scanner & SCANLAB IntelliScan 14 & Rayleigh length & $1.43 \mathrm{~mm}$ \\
\hline Focal length & $100 \mathrm{~mm}$ & & \\
\hline
\end{tabular}

Three samples were welded per parameter. Apart from the parameters laser power, feed rate and number of passes, the line energy was used to compare the welding seams. It is defined as:

$$
E=\frac{P}{v} * n
$$

where $\mathrm{P}$ is the laser power, $\mathrm{v}$ the feed rate and $\mathrm{n}$ the number of passes. Thin sections were made perpendicular to the welding seam with a thickness of $14 \mu \mathrm{m}$ using a rotary microtome RM2255 (Leica Biosystems Nussloch GmbH, Nussloch, Germany). The thin sections were then analysed using a polarisation microscope Axio Imager A2.m of the company Carl Zeiss Microscopy GmbH, Jena, Germany. Both the height and width of the HAZ were measured (see Fig. 5).

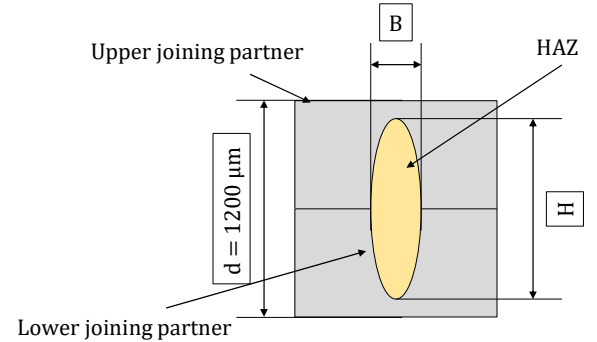

Fig.5 Analysation of the HAZ dimensions

\section{Experimental results}

3.1 Influence of focal position on the HAZ

Starting from the focal position at the interface $(\mathrm{z}=0)$, welding trials were carried out at $\mathrm{E}=2.5 \mathrm{~J} / \mathrm{mm}$ with $\mathrm{P}=22.5 \mathrm{~W}, \mathrm{v}=450 \mathrm{~mm} / \mathrm{s}$ and $\mathrm{n}=50$. The focal position was then moved in $1 \mathrm{~mm}$ steps along the beam propagation direction $(\mathrm{z}>0)$.

As it can be seen in Figure 6, the focal position has only minor influence on the HAZ dimensions. The focal position was varied at a distance of $9 \mathrm{~mm}$ in total which is about 6 times the Rayleigh length of the laser beam. The reason why the focal distance was only varied in positive z-direction is that in negative z-direction, the beam focus would be moved towards the surface of the upper joining partner. This in turn can cause burnings due to the high intensities at the surface. By moving the focal position in positive z-direction on the other hand, the intensity decreases on the surface of the upper joining partner.

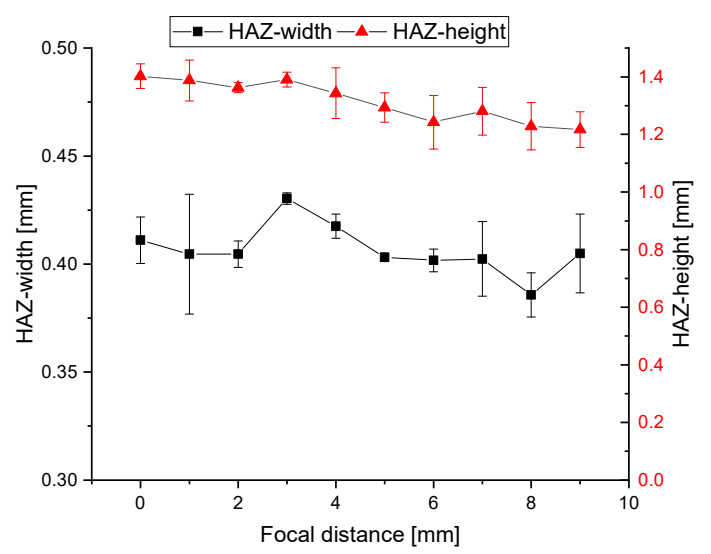

Fig.6 HAZ dimensions at different focal positions

The results show that due to heat accumulation, the HAZ is formed in the centre of the sample. If both joining partners have the same thickness, the HAZ is therefore always located in the interface. Hence, a variation of the focal position has only a slight effect on the HAZ dimensions. This is an advantage as it reduces the time to set up the welding process. In contour welding with high NA optics on the other hand, an unprecise positioning of the beam focus can lead to a HAZ outside of the interface.

\subsection{Influence of the number of passes at constant line energy on the $\mathrm{HAZ}$}

In a next step, the number of passes were increased at a constant line energy of $2.5 \mathrm{~J} / \mathrm{mm}$ and laser power of $22.5 \mathrm{~W}$. 
Therefore, the feed rate was increased with increasing number of passes in order to keep E constant (see equation 5).

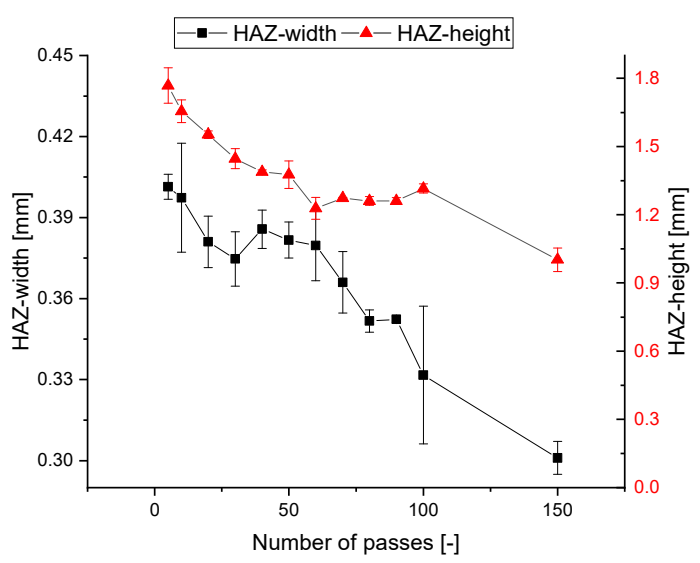

Fig.7 HAZ dimensions at different number of passes at constant line energy

Fig. 7 shows the results for the HAZ width and height. The seam length was $16 \mathrm{~mm}$. It can be seen that with increasing number of passes, the vertical extent of the HAZ decreases. Furthermore, the HAZ width also decreases. At $\mathrm{n}=150$, the HAZ height is $1 \mathrm{~mm}$ compared to $1.7 \mathrm{~mm}$ at $\mathrm{n}=1$ which leads to a decrease of $43.4 \%$. On the other hand, the HAZ width is reduced from $0.4 \mathrm{~mm}$ at $\mathrm{n}=1$ to $0.3 \mathrm{~mm}$ at $\mathrm{n}=150$ which makes a decrease of $33.2 \%$. The microscopic measurements can be seen in Figure 8.

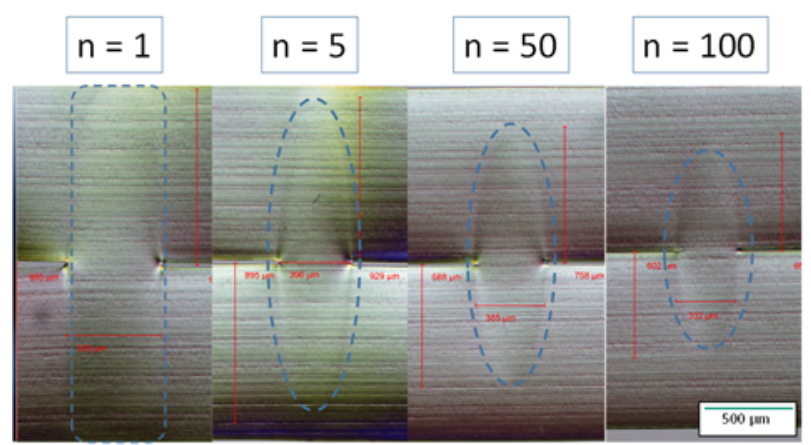

Fig.8 Reduction of the HAZ with increasing number of passes

\subsection{Influence of the seam distance on the HAZ}

In case of microfluidic devices, the encapsulation of the microchannels leads to high seam densities in the device. This in turn can cause an increased thermal stress on the device during the welding process which in turn can lead to the formation of cracks and distortions. For example, when a weld seam is created, the surrounding material is also heated. This can influence the geometry of the HAZ of the subsequently produced welding seams. In order to investigate this interaction effect, eight weld seams with defined distances $\Delta \mathrm{y}$ were created. The distances were varied between $1 \mathrm{~mm}$ and $1.9 \mathrm{~mm}$. Figure 9 shows an example of the cross section of the welding seams at a distance of $1.6 \mathrm{~mm}$.

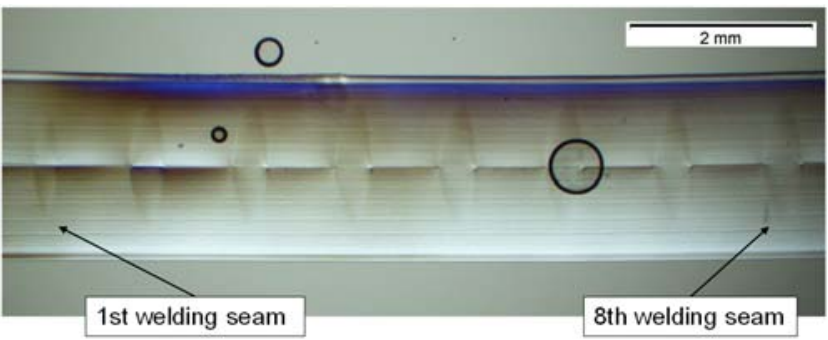

Fig.9 HAZ at a seam distance of $1.6 \mathrm{~mm}$

It can be seen that the first welding seam has the smallest vertical and horizontal expansion, while the subsequent welds are larger. The reason is that at the beginning of the first welding process the temperature of the sample material corresponds to the room temperature. The welding process causes the surrounding material to heat up. Due to the higher initial temperature, a higher maximum temperature is reached in the component while the welding parameters remain constant. This in turn leads to a larger weld seam. The measured widths of the HAZ are shown in Figure 10 for $\Delta \mathrm{y}=1 \mathrm{~mm}, 1.3 \mathrm{~mm}, 1.6 \mathrm{~mm}$ and $1.9 \mathrm{~mm}$.

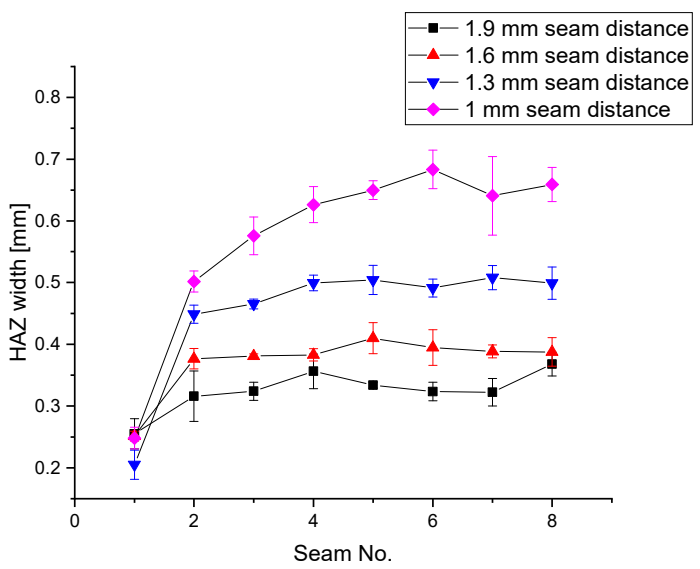

Fig.10 HAZ width at different seam distances

For the first seam, no significant differences in the weld geometry were observed for all investigated line distances, whilst an increase in the width of the HAZ was measured for the welding seams No. 2 to No. 8 . At a distance of $1.9 \mathrm{~mm}$, the increase is the smallest, whilst at a distance of $1 \mathrm{~mm}$ it is the largest. The reason for this is that as the seam distance decreases, the interaction effects are stronger and the initial temperature for weld seams No. 2 to No. 8 is higher. It can also be seen that the weld widths are approaching a limit value. This is achieved earlier the higher the seam distances are. For example, this limit value is reached at $\Delta y=1.6$ and $\Delta y=1.9 \mathrm{~mm}$ from the 2nd seam onwards. At $\Delta y=1.3 \mathrm{~mm}$ and $\Delta y=1 \mathrm{~mm}$, the limit values are not reached until the 4th or 6th seam respectively. 


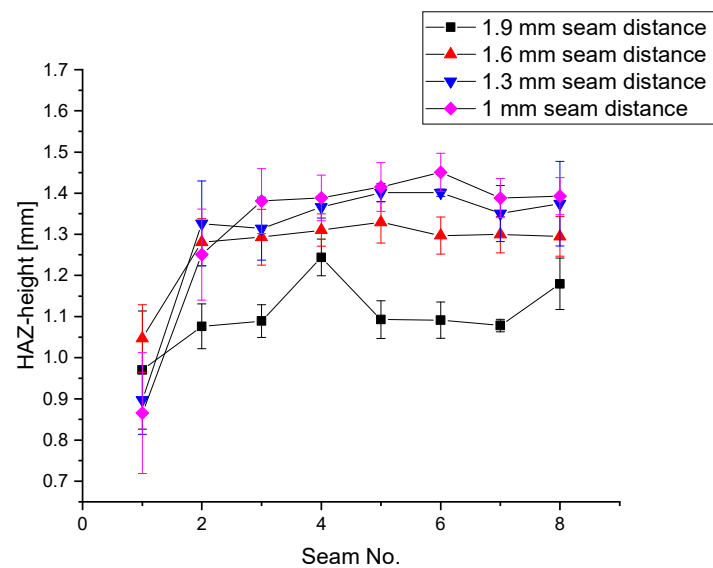

Fig.11 HAZ height at different seam distances

The line distances have the same effect on the formation of the height of the HAZ (see Fig. 11). Similarly, the HAZ height increases with decreasing seam distance. For the line distances $1.3 \mathrm{~mm}, 1.6 \mathrm{~mm}$ and $1.9 \mathrm{~mm}$, the HAZ height reaches a limit value from the 2 nd seam onwards. At a line distance of $1 \mathrm{~mm}$, this limit value is reached from the $3 \mathrm{rd}$ seam onwards. In contrast to the HAZ width, the HAZ height is subject to stronger fluctuations. For example, the HAZ height of the first seam varies between 0.85 and $1.05 \mathrm{~mm}$. Furthermore, the HAZ heights at $\Delta y=1-1.6 \mathrm{~mm}$ are close together with values between 1.25 and $1.4 \mathrm{~mm}$.

\subsection{Influence of the cooling time on the HAZ}

In the previous chapter it was shown that after the welding of the first seam, the increased material temperature causes an influence on the geometries of the following seams. This influence decreases with higher seam distances. This undesirable interaction can also be avoided by increasing the cooling time. In order to investigate the influence of the cooling time, two weld seams with a line distance of 0.45 $\mathrm{mm}$ to each other were produced. The second seam was created after a time interval of $0,5,15,30$ and $60 \mathrm{~s}$.

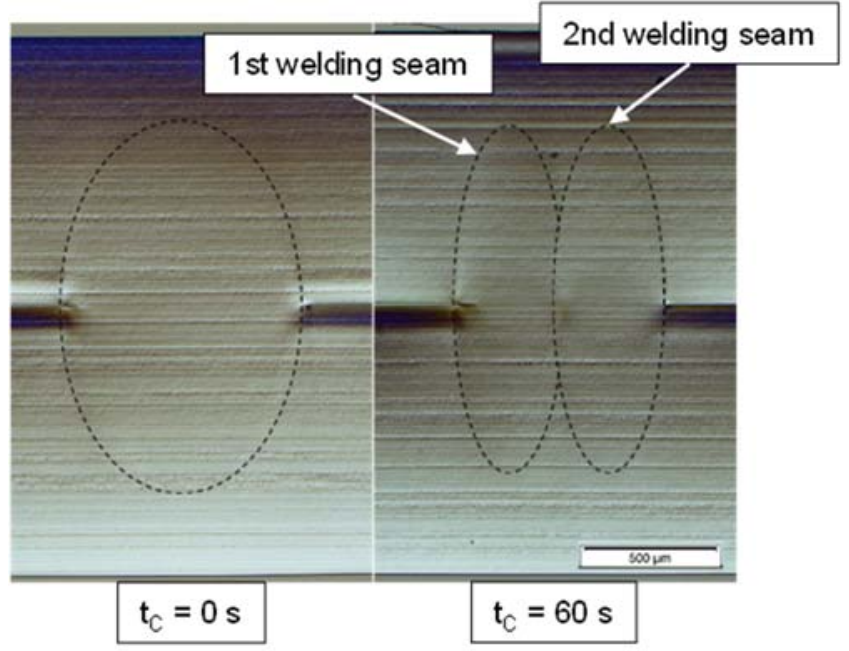

Fig.12 HAZ at a cooling time of $0 \mathrm{~s}$ (left) and $60 \mathrm{~s}$ (right)

Figure 12 shows the HAZ for a cooling time of $0 \mathrm{~s}$ and $60 \mathrm{~s}$. The laser power is $28 \mathrm{~W}$, the feed rate is $450 \mathrm{~mm} / \mathrm{s}$ and the number of passes is 50 . At a cooling time of $0 \mathrm{~s}$, there is no clear boundary between the two weld seams. The reason for this is that the material did not cool down after the generation of the first seam and the melt has therefore not yet solidified. Instead of two separate welding seams, a single elliptical seam is formed. On the right hand side, however, the second weld seam was created after a cooling time of $60 \mathrm{~s}$. Due to the longer cooling time, the melt of the first seam solidified completely before the second seam was created. As a consequence, two separate HAZ are recognizable here, which overlap slightly in their horizontal expansion. The width and height of the combined HAZ were measured and summarised in Figure 13.

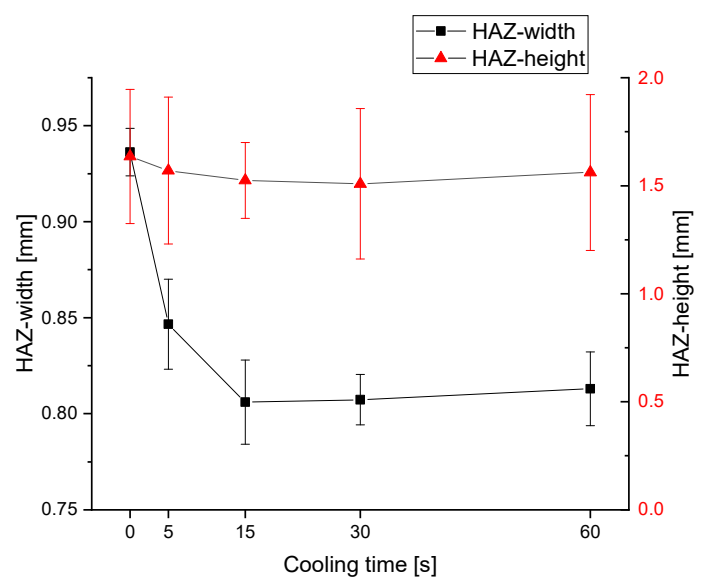

Fig.13 HAZ dimensions at different cooling times

With a cooling time of $0 \mathrm{~s}$, the largest HAZ with a width of $0.93 \mathrm{~mm}$ and a height of $1.63 \mathrm{~mm}$ could be measured. This was due to the higher initial temperature in the material after the first weld. Similar to the experiments in Chapter 3.3, a larger HAZ is generated at the same line energy. With a cooling time of $5 \mathrm{~s}$, the width is reduced to $0.84 \mathrm{~mm}$. From a cooling time of $15 \mathrm{~s}$ onwards, the width reaches its lower limit which does not decrease significantly with higher cooling times. This indicates that from this cooling time on, the melt of the first weld seam is completely solidified. On the other hand, the height of the HAZ remains constant with longer cooling times. On top of that, a higher deviation of the results is also noticeable here. One possible reason could be that the temperature of the material after creating the first welding seam is higher in the joining interface than on the outside due to heat accumulation. Because of that, a higher cooling time would have a lower influence on the vertical propagation of the HAZ.

\section{Segmentation of the seam geometry and determina- tion of an irradiation sequence}

In order to avoid heat accumulation and thus a widening of the weld seam during the welding process, a test contour was divided into several segments. By defining an irradiation sequence for each segment, an even distribution of the heat is ensured. The test contour is shown in Figure 14. In a first trial, the segments are welded one after another. The irradiation sequence is indicated by numbers. After that, the irradiation order of the segments was set so that the distance between two successive segments is maximum. Each segment has a length of $15 \mathrm{~mm}$, the welding parameters are a power of $28 \mathrm{~W}$, a feed rate of $450 \mathrm{~mm} / \mathrm{s}$ and 50 passes. 


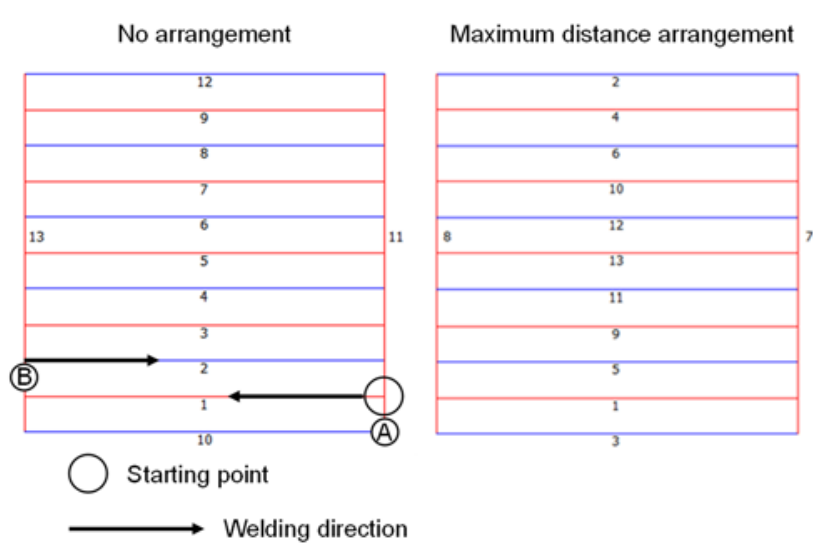

Fig.14 Welding segments without arrangement (left) and a maximum distance arrangement (right). The numbers show the irradiation order.

The areas $\mathrm{A}$ and $\mathrm{B}$ will be examined in more detail below. In case that no irradiation sequence is defined in area $A$, the end point of a segment is directly connected to the start point of the following segment (see Fig. 15, left side). The preheating of the material clearly shows that the welding seam width of the following segment is larger at the beginning and decreases again at a greater distance from the starting point. On the other hand, if the maximum distance arrangement is applied, no broadening of the weld seam can be observed (see Fig. 15, right side).

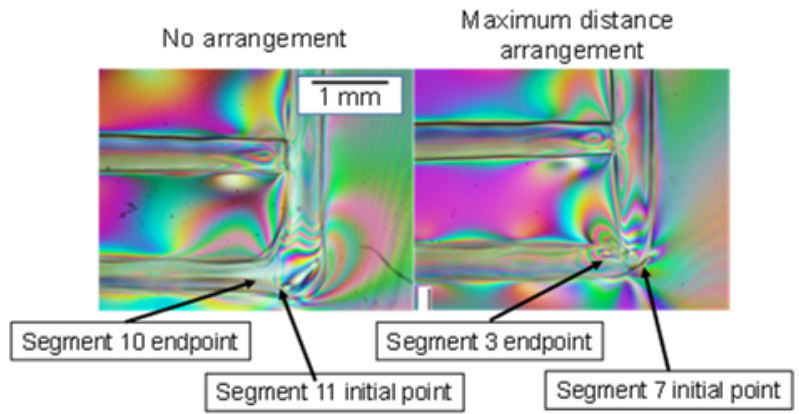

Fig.15 Welding segments without arrangement (left) and a maximum distance arrangement (right) for area A.

In area B (see Fig.16), the distance between the end point of segment 1 and the start point of segment 2 is $1 \mathrm{~mm}$. If no irradiation sequence is defined, the width of the weld increases in segment 2 . The width here is $462 \mu \mathrm{m}$ while the seam width in segment 1 is $360 \mu \mathrm{m}$. In the case of a maximum distance arrangement, segment 2 is now welded in fifth place, which allows the surrounding material to cool down in between. As a result, the seam width is almost the same. This is $423 \mu \mathrm{m}$ for segment 1 and $438 \mu \mathrm{m}$ for segment 5 .

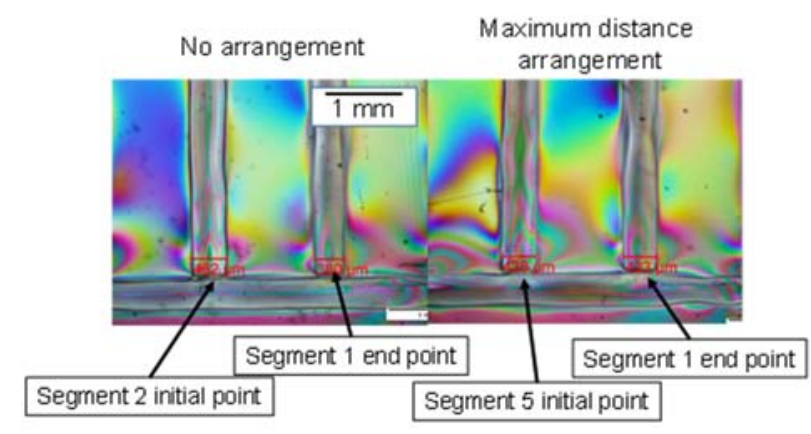

Fig.16 Welding segments without arrangement (left) and a maximum distance arrangement (right) for area B.

\section{Encapsulation of a microfluidic demonstrator part}

The process understanding developed in the context of this work was applied to weld a microfluidic test component made of PC which was provided by microfluidic ChipShop $\mathrm{GmbH}$, Jena, Germany. The component consists of a microfluidic basic body with four channel structures. The four channels form reaction chambers with a volume of $50 \mu \mathrm{l}$ each. The basic body is encapsulated with a cover plate. Figure 17 shows a welding seam which was generated at the edge of the channel.

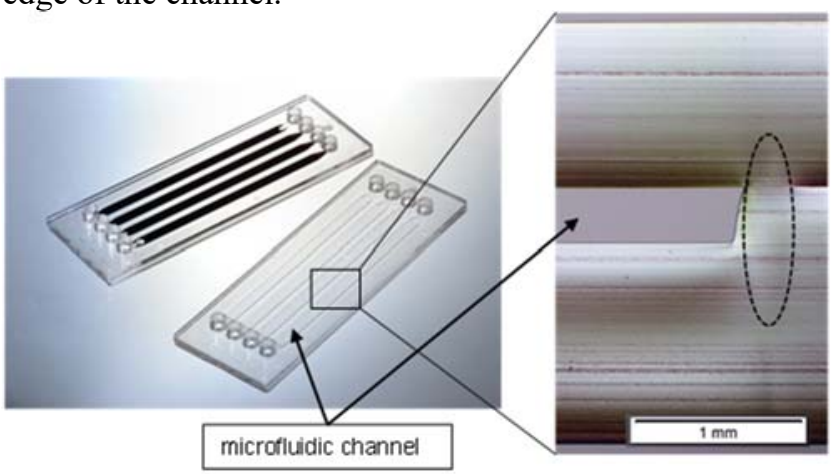

Fig.17 Welded microfluidic demonstrator part

It can be seen that the base body is thicker than the cover plate. Therefore, the highest HAZ width is not located in the joining zone, but in the base body. As test parameters, a power of $28 \mathrm{~W}$, a feed rate of $450 \mathrm{~mm} / \mathrm{s}$ and 50 passes were set. The maximum distance arrangement was set in order to enable an even distribution of heat and cooling of the seam segments. Ink was pumped through the channels for control purposes. It can be seen that the channels were encapsulated media-tight and no ink escapes from the channels.

\section{Conclusion}

Within the scope of the presented work, it was shown for absorber-free LTW that a precise melting can be achieved by using a quasi-simultaneous welding technique. Furhtermore, the focal position plays a minor role in this case, since the heat accumulates in the centre of the component. Due to the high seam densities in microfluidic components, the influence of weld seam distances and cooling time on the formation of the HAZ was investigated. The process understanding developed within the scope of the work was applied to weld a microfluidic test component. The channel structures could be encapsulated media-tight. Furthermore, a determination of the irradiation sequence of the seam segments can counteract heat accumulation and thus a further extension of the welding seams. However, in order to be better 
able to determine an optimum irradiation sequence, further studies should be focused on the determination of the irradiation sequence on the basis of a measured temperature signal. Possible approaches here are measurements by means of thermographic cameras or pyrometers.

\section{Acknowledgments}

This project is co-funded by the European Regional Development Fund and the German federal state of North Rhine Westphalia (EFRE-0800726).

The authors would like to thank microfluidic ChipShop $\mathrm{GmbH}$, Jena, Germany for providing the microfluidic specimens.

\section{References}

[1] E. Haberstroh, W.-M. Hoffmann, R. Poprawe, and F. Sari: Microsys. Tech., 12, (2006) 7.

[2] D. Grewell and A. Benatar: Int. Polymer Proc., 22, (2007) 1.

[3] V. Mamuschkin, C. Engelmann and A. Olowinsky: Phys. Proc., 83, (2016).
[4] I. Jones: Assembly Automation, 22, (2002), 2.

[5] I. Mingareev, F. Weirauch, A. Olowinsky, L. Shah, P. Kadwani, M. Richardson: Opt. \& Las. Techn., 44, (2012) 7.

[6] S. Ruotsalainen, P. Laakso, V. Kujanpää: Phys. Proc., 78, (2015).

[7] M. Brosda, A. Olowinsky, A. Pelzer: Phys. Proc., 83, (2016).

[8] V. Mamuschkin, M. Aden, A. Olowinsky: Las. Man. Mat. Proc., (2019)

[9] P. Laakso, S. Ruotsalainen, G. Otto, A. Olowinsky, V. Kujanpää: Proc. ICALEO (2013).

[10] V. Mamuschkin, A. Haeusler, C. Engelmann, A. Olowinsky, H. Aehling: J. Las. Appl., 29, (2017) 2.

[11] V. Mamuschkin, A. Olowinsky, K. van der Straeten, C. Engelmann: Proc. SPIE, (2015).

[12] M. Aden, A. Gillner, G. Otto, V. Mamuschkin: Join. Plast., 12, (2018).

[13] A. Schmailzl, S. Hierl, M. Schmidt: Phys. Proc., 83, (2016).

(Received: May 18, 2019, Accepted: December 24, 2019) 